\title{
Review
}

\section{근본원인분석 수행을 위한 지침}

\section{Guidelines for Performing Root Cause Analysis}

- 이현정', 최은영', 옥민수 ${ }^{2}$, 이상일1

Hyeon-Jeong Lee ${ }^{1}$, Eun-Young Choi ${ }^{1}$, Min-Su Ock², Sang-Il Lee ${ }^{1}$

- 울산대학교 의과대학 예방의학교실 ${ }^{1}$, 울산대학교 의과대학 울산대학교병원 예방의학과 ${ }^{2}$

Department of Preventive Medicine, College of Medicine, University of Ulsan'

Department of Preventive Medicine, Ulsan University Hospital, College of Medicine, University of Ulsan²

- 교신저자 : 이 상 일

주소 : 05505, 서울 송파구 올림픽로 43길 88 울산대학교 의과대학 예방의학교실

전화 : 02-3010-4284

팩스번호 : 02-477-2898

전자우편주소 : sleemddaamc.seoul.kr

Correspondence : Sang-Il Lee

Address : Department of Preventive Medicine, University of Ulsan College of Medicine, 88 Olympic-ro 43-gil,

Songpa-gu, Seoul 05505, Korea

Tel : +82-2-3010-4284

FAX : +82-2-477-2898

E-mail : sleemddamc.seoul.kr

Funding : 한국보건산업진흥원 (Fund No. HI16C0173) Conflict of Interest : None
Received : Jun.01.2017

Revised : Jun.21.2017

Accepted : Jun.21.2017 


\section{Review}

\section{Abstract}

Root cause analysis (RCA) is systematic process for identifying contributing factors and root causes. It detects system-level vulnerabilities and prevents them from occurring in the future. In many countries, RCA guidelines have been developed and used for these purposes, and various practical tools are suggested according to stages of RCA implementation. In Korea, adverse events occur in 7.2-8.3 percent of inpatients according to studies conducted in hospitals. However, frontline staffs are suffering from lack of knowledge about RCA implementation. This study introduces RCA guidelines that may be used in hospitals to improve the quality of medical care and patient safety.

\section{Key words}

Patient safety, Root cause analysis, Guideline 


\section{I . 서론}

환자안전사고란 환자에게 보건의료서비스를 제공 하는 과정에서 환자의 생명, 신체, 정신에 대한 손 상 또는 부작용의 위해가 발생하였거나 발생할 우 려가 있는 사고를 말한다[1]. 우리나라의 환자안전 현황에 대해 전국적인 조사가 수행된 사례가 없다. 다만 일부 병원을 대상으로 수행한 연구에 따르면 유해사례(adverse event)의 발생확률은 $7.2 \%$ 내지 $8.3 \%$ 로 나타났으며[2] 이는 국외의 체계적 문헌고 찰 결과에서 나타난 $9.2 \%$ 와 유사한 수준이었다[3]. 국내 연구 결과를 2015년 건강보험통계연보[4]의 입원 진료실인원에 적용할 경우 2016년에 입원했던 환자 중 약 48 55만 명이 유해사례를 경험했던 것 으로 추정해 볼 수 있다.

이에 우리나라에서는 환자를 보호하고 의료의 질 을 향상시키기 위한 목적으로 2015년에 환자안전 법을 제정하였다. 이 법에는 보건복지부장관이 환 자안전사고 보고 - 학습시스템을 구축하여 운영해야 한다는 조항이 있다. 하지만 아직까지 국가 수준에 서 환자안전사고를 총괄적으로 관리하는 외부 보고 시스템이 없으며[5], 의료기관에서 개별적으로 내부 보고시스템을 운영하고 있는 실정이다. 국내 93 개 의 병원을 대상으로 실시한 연구에 따르면 92 개의 병원이 보고시스템을 자체적으로 운영하고 있었다 [6]. 보고 · 학습시스템의 목적은 환자안전사고의 보 고에 그치는 것이 아니라 보고된 자료를 분석하여 학습한 후 향후 발생 가능한 환자안전사고를 미연 에 방지하는 것이다.

이와 관련하여 환자안전법은 환자안전 전담인력 의 업무 중 하나로써 환자안전사고에 대한 분석 을 규정하고 있다. 의료기관평가인증원의 환자안전 법 운영 매뉴얼[7]에도 환자안전법 상의 환자안전사 고 정보 분석 업무와 관련하여 근본원인분석(root cause analysis, RCA)의 실시를 제시하고 있다. 그 러나 Shin [6]의 연구에 따르면 환자안전사고에 대 해 $\mathrm{RCA}$ 를 실시하는 병원은 조사 대상 병원의 $7.6 \%$ 에 불과하였다. 국내의 급성기 의료기관의 인증준 비 전담자를 대상으로 한 설문조사에서 응답자의 $38.3 \%$ 가 RCA를 실시하지 않는 것으로 나타났는데, 특히 400 병상 이하인 경우는 $64.1 \%$ 가 RCA를 하지 않고 있었다[8]. 이 조사 결과에 따르면 $\mathrm{RCA}$ 를 실 시하지 않는 이유는 전체 응답자 중 $17.6 \%$, 특히 400 병상 이하인 경우 $24.2 \%$ 에서 RCA에 대한 지식 부족이 부족하기 때문이었다. 즉, 개별 의료기관이 보고시스템을 운영하고 있어도 그 안에서 $\mathrm{RCA}$ 를 원 활히 수행하지 못하고 있는 것으로 볼 수 있다.

$\mathrm{RCA}$ 는 원자력 발전, 항공 등의 산업 시스템 분 야에서 개발한 방법으로 의료분야에서는 미국의 보 훈청(Veterans Administration)과 미국의 대표적인 의료기관 인증기관인 더 조인트 커미션(The Joint Commission, TJC)이 1990년대 중반에 도입하였다 [9]. RCA의 주요 핵심질문은 무슨 일이 일어났는지, 그 일이 왜 일어났는지, 사건 재발을 예방하기 위해 무엇을 해야 하는가이다. $\mathrm{RCA}$ 는 사건의 표면적이 고 직접적인 원인보다는 사건 아래에 내재되어 있 는 근본원인을 발견해 나가는 절차이다. 또한 이미 일어난 과거의 사건에 대해 개인 수준의 책임 소재 를 밝히기 보다는 시스템 수준에서의 취약성을 개 선시켜 미래에 발생할 가능성이 있는 사건을 예방 하고자 하는 목적 지향적이고 체계적인 절차이다.

우리나라는 국가 수준의 보고시스템을 갖추고 있 지 않은 상태에서 개별 의료기관이 내부 보고시 스템을 운영하고 있다. 그러나 일부 의료기관에서 만 보고된 사건에 대해 원인분석을 하고 있었으며, $\mathrm{RCA}$ 를 하지 않는 주요한 이유 중 하나는 $\mathrm{RCA}$ 방 법을 제대로 알지 못하기 때문이었다. 그럼에도 국 내에는 $\mathrm{RCA}$ 수행에 대한 지침이나 관련 연구가 부 


\section{Review}

\section{근본원인분석 수행을 위한 지침}

족한 실정이다. 이에 이 종설에서는 미국, 영국, 캐 나다, 일본에서 사용하고 있는 $\mathrm{RCA}$ 지침을 정리하 여 소개하고자 한다.

\section{II. 본론}

\section{1. 미국 더 조인트 커미션의 지침}

$\mathrm{TJC}$ 에서는 $\mathrm{RCA}$ 절차를 21 단계로 나누어 설명 하는 지침을 발표하였다[10]. 이 지침에서는 이미 발생한 적신호사건(sentinel event)이나 거의 발생 할 뻔 한 적신호사건의 기반원인을 알아보기 위해 서 사용하는 $\mathrm{RCA}$ 에 초점을 맞추고 있다. $\mathrm{TJC}$ 의 지침에 의하면 적신호사건은 예상치 못한 상태에 서 발생한 사건으로써 사망이나 심각한 물리적, 심
리적 상해 또는 그 위험성을 수반하는 사건을 의 미한다. 또한 $\mathrm{RCA}$ 는 성과면에서 변이가 생기도록 하는 내재적인 기본 요인과 원인이 되는 요인을 규 명하는 과정이라고 하였다. 근본원인은 성과가 기 대에 미치지 못한 상황 또는 과오가 일어난 가장 근본적인 원인으로써 시스템적인 원인이라고 보았 다. $\mathrm{TJC}$ 는 $\mathrm{RCA}$ 를 통해 개인과 결과가 아니라 시 스템과 과정에 초점을 두고 분석하여 이에 대한 개 선점을 찾는 것이 중요하며, 여러 의료기관 등 조 직 간에 $\mathrm{RCA}$ 를 통해 얻은 정보를 공유하는 것이 필요하다고 하였다.

$\mathrm{TJC}$ 의 지침은 RCA 준비, 근접원인 규명, 근본원 인 규명, 개선활동의 설계 및 도입으로 나누어 RCA 수행 단계를 제시하고 있으며 각 단계마다 사용할 수 있는 도구와 활용할 수 있는 문서 양식 등을 구 체적으로 제공하고 있다(Table 1).

Table 1. Process for root cause analysis of The Joint Commission

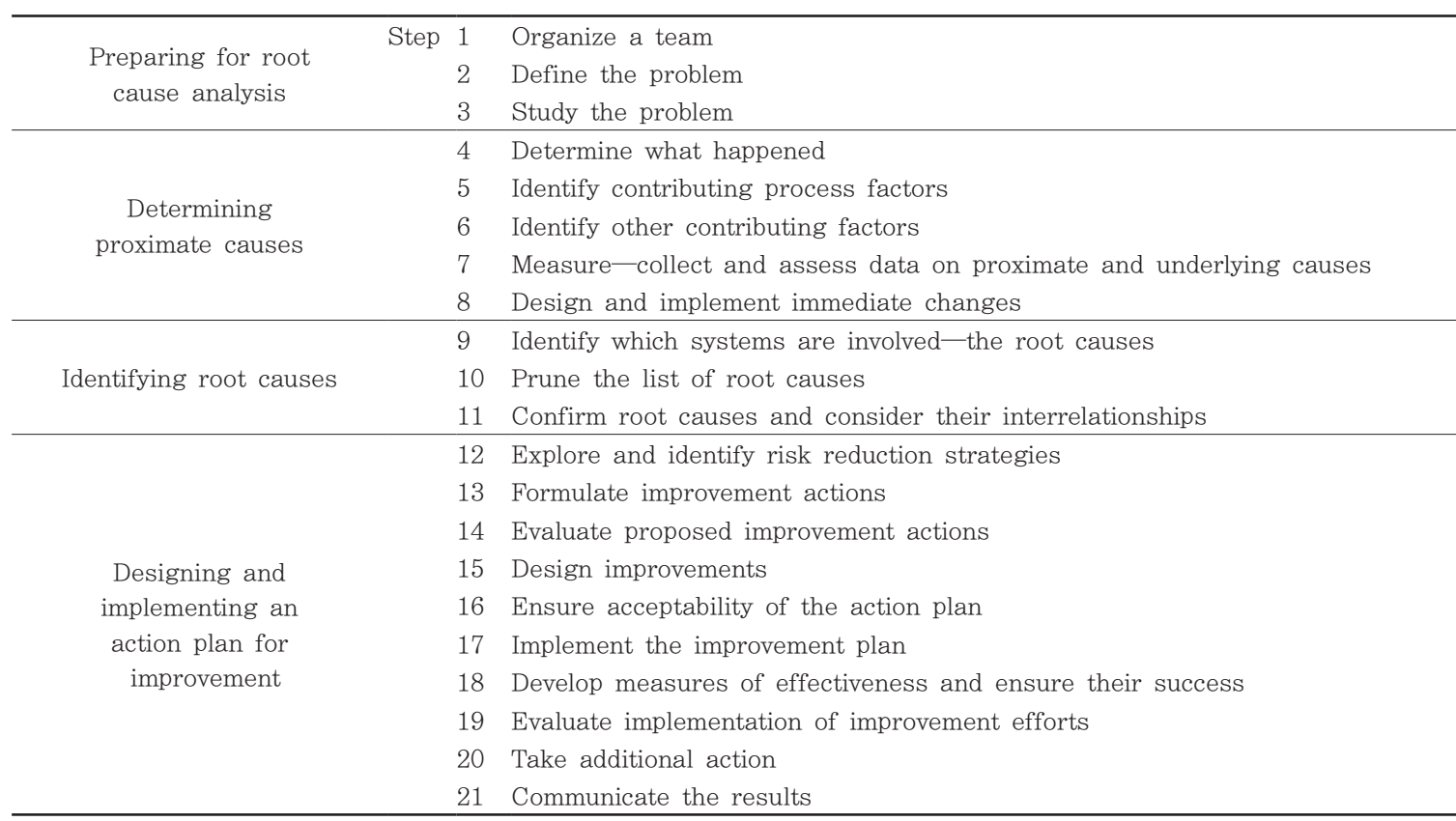

Source: Parker J. editor. The Joint commission. Root Cause Analysis in Health Care: Tools and Techniques. 5th ed. Illinois, USA: Oak Brook; 2015. 
$\mathrm{RCA}$ 준비 단계는 $\mathrm{RCA}$ 를 수행할 팀을 조직하고, 문 제를 정의하고, 문제를 분석하기 위해 데이터를 수집 하는 단계이다. 핵심적인 팀 구성원의 요건, 인터뷰 및 정보 수집 방법, 정보 기록 방법을 제공하고 있다. 근접원인 규명 단계에서는 사건의 발생 과정에 대한 기술 방법과 사건에 대해 가장 분명하거나 직접적으 로 영향을 주는 기반원인을 확인하는 방법에 대해 설 명하고 있다. 확인한 여러 원인에 대한 데이터를 수 집하고 측정하는 방법과 근접원인을 확인한 초기 단 계에서 즉각적인 변화를 위해 할 수 있는 수행 방법 에 대해서도 설명한다. 근본원인 규명 단계에서는 개 인의 실수보다는 시스템의 문제에 초점을 맞추어 깊 이 있게 탐색하는 과정, 이때 사용할 수 있는 질문, 가 능한 모든 인과요인을 찾기 위해 사용할 수 있는 분 류체계, 찾아낸 원인 중 근본원인을 확인하기 위한 방 법을 제시하고 있다. 마지막으로 개선활동 계획의 설 계 및 도입 단계에서는 위험을 감소시키고 오류를 방 지하기 위한 구체적 전략, 개선행위 수립 및 우선순위 부여 방법, 개선행위 평가 항목, 개선활동에 대한 보 고 지침을 자세히 제공하고 있다.

$\mathrm{TJC}$ 는 $\mathrm{RCA}$ 시행 시 모든 단계를 다 거쳐야 할 필 요는 없으며 한 번에 여러 단계를 동시에 수행하거나 다음 단계로 넘어가기 전에 이전 단계로 돌아갈 수도 있다고 하였다. 그리고 $\mathrm{RCA}$ 를 실시하는 조직의 상황 에 맞게 조절하여 사용할 수 있다고 설명하고 있다. 또한 이 지침에서는 RCA에서 사용할 수 있는 도구로 써 친화도, 인과관계도, 오류형태 및 영향분석, 물고 기뼈도식, 순서도, 간트차트 등의 사용방법과 이러한 도구를 적용할 수 있는 단계를 제시해주고 있다. 이와 함께 $\mathrm{RCA}$ 를 적용한 구체적인 사례를 제공하고 있다.

\section{2. 미국 국가환자안전센터의 지침}

미국 보훈청은 산하 의료기관에서 수행한 $\mathrm{RCA}$ 를
보고하도록 하고 있으며, 국가환자안전센터(National Center for Patient Safety, NCPS)의 홈페이지에 $\mathrm{RCA}$ 를 위한 지침인 근본원인분석 단계별 지침(Root Cause Analysis Step-by-step Guide)과 RCA 수 행 시 필요한 자료를 제공하고 있다[11]. 이 지침은 NCPS의 환자안전 정보시스템(Patient Safety Information System) 환경을 기준으로 설명하고 있 다. 이 지침에서는 모든 유해사례뿐만 아니라 검토 가 필요하다고 판단한 근접오류(near miss)에도 적 용하며, 개인의 잘못이 아니라 설계나 시스템의 실 패에 초점을 맞추어야 한다고 설명하고 있다. 그래 서 $\mathrm{RCA}$ 를 위한 핵심질문에는 무엇이 왜 발생했으 며, 이러한 일이 다시 발생하는 것을 예방하기 위해 취해야 할 행동에 대한 질문이 있으나 누구(who)에 대한 질문은 없다.

$\mathrm{NCPS}$ 는 RCA 수행 방법을 21단계로 제시하고 있 다. NCPS의 지침은 $\mathrm{TJC}$ 의 지침과 유사하게 구성되 어 있는데 큰 범주로 나누어보면 RCA 준비, $\mathrm{RCA}$ 수행, 개선활동 수행 계획 수립 및 $\mathrm{RCA}$ 결과 공유 로 구성되어 있다. $\mathrm{RCA}$ 준비 단계는 $\mathrm{RCA}$ 수행 여 부 결정, 필요한 경우 즉각적인 행동 수행, 선언문 (charter) 작성 및 RCA 팀 구성으로 이루어져 있다. $\mathrm{RCA}$ 수행 단계는 사건순서도 초안 작성, 부족한 정 보 확인, 필요한 정보의 구체화, 인터뷰 및 관련 자 료 검토를 통한 사실 확인, 정보 합성, 사건순서도 최 종 확정, 근본원인 및 기여요인 확인으로 진행된다. $\mathrm{RCA}$ 수행 후에는 유사한 사례에 대해 과거에 수행 하였던 $\mathrm{RCA}$ 의 개선활동 확인, 개선활동 계획 수립, $\mathrm{RCA}$ 및 개선활동 계획 및 평가 계획의 공유, $\mathrm{NCPS}$ 에 $\mathrm{RCA}$ 결과 제출 전 개인정보 삭제, $\mathrm{RCA}$ 에 소요된 비용 및 인당 시간 산정, 최종 완료된 $\mathrm{RCA}$ 에 대한 담당자들의 종료 서명까지 포함하고 있다.

특히 NCPS 지침은 모든 사건에 대해 안전평가코 드(safety assessment code, SAC) 매트릭스(ma- 


\section{Review}

\section{근본원인분석 수행을 위한 지침}

trix)라는 도구를 이용하여 $\mathrm{SAC}$ 점수를 1 3점으로 부여한 후 여기서 고위험인 3점으로 측정되는 경우 $\mathrm{RCA}$ 를 수행하도록 하고 있다(Table 2). SAC 매트릭 스는 환자의 상태에 따른 중증도(severity)와 해당 의 료기관에서 동일한 사건이 발생했던 빈도에 따라 고 위험(3점), 중등도 위험(2점), 저위험(1점)으로 나누 어 사건을 분류한다. SAC 매트릭스에서 환자 상태 의 중등도는 유해사례로 인해 사망이나 영구적으로 주요한 기능 손실이 발생한 경우(catastrophic), 영구 적으로 신체 기능의 감소가 발생한 경우(major), 병 원 재원기간이 증가하거나 제공해야할 의료서비스의 수준이 높아진 경우(moderate), 손상이나 재원기간 의 증가가 없는 경우(minor)로 구분한다. 사건 발생 빈도는 1년에 몇 번 발생하는 경우(frequent), 1 2 년에 몇 번 발생하는 경우(occasional), 2 5년에 가 끔 발생하는 경우(uncommon), 5 30년에 가끔 발생
하는 경우(remote)로 구분한다.

또한 NCPS 지침은 인과의 법칙(rules of causation) 을 아래와 같이 제시하고 있는데, 이는 사건이 근본 원인 또는 기여요인과 어떻게 관련되어 있는지를 기 술하는 근본원인진술문(root cause statement)을 작 성할 때 사용할 수 있는 지침이다.

- 원인과 영향에 대해 명확하게 연결하여 서술한다. - 원인을 나타내는 진술문에는 부정적이거나 비판적 인 단어를 사용하지 않는다.

- 각각의 인적 오류에는 반드시 선행되는 시스템 수 준의 원인이 있다.

· 정책과 절차의 위반은 근본원인이 아니며, 반드시 선행되는 시스템 수준의 원인이 있다.

- 행동을 하지 않은 것은 행동할 것에 대한 의무가 존재할 때만 원인이 된다.

Table 2. Safety assessment code matrix

\begin{tabular}{cccccc}
\hline & & & Severity & & \\
\cline { 3 - 5 } & & Catastrophic & Major & Moderate & Minor \\
\hline \multirow{3}{*}{ Probability } & Frequent & 3 & 3 & 2 & 1 \\
& Occasional & 3 & 2 & 1 & 1 \\
& Uncommon & 3 & 2 & 1 & 1 \\
\hline
\end{tabular}

Source: Veterans Administration National Center for Patient Safety. Root Cause Analysis [cited 2017 Apr 10]. Available from: https://www.patientsafety.va.gov/professionals/onthejob/rca.asp.

\section{3. 미국환자안전재단의 근본원인분석과 행동}

미국환자안전재단(National Patient Safety Foundation, NPSF)은 닥터스 컴퍼니 재단(Doctors Company Foundation)의 지원을 받아 전문가 및 이해관 계자들의 협력 하에 $\mathrm{RCA}$ 프로세스를 향상시키기 위 한 방법으로 근본원인분석과 행동(RCA and Action, $\left.\mathrm{RCA}^{2}\right)$ 을 개발하였다[12]. NPSF는 RCA가 하나의 근
본원인을 연상시키고 이 용어로 인해서 $\mathrm{RCA}$ 의 목적 이 분석에 한정된다고 보았다. $\mathrm{RCA}$ 의 의미를 제한하 지 않고 RCA 후 시스템 개선을 위한 활동수행을 강 조하기 위해 $\mathrm{RCA}^{2}$ 라는 용어를 사용하고 있다. $\mathrm{RCA}^{2}$ 지침은 위해(harm)를 입을 가능성으로써 유해사례 전에 존재하는 조건적인 상태를 해저드(hazard)라 고 하였고, 해저드로 인해 예상되는 손실의 측정치 를 리스크(risk)라고 하였다. 이때 리스크는 손실의 
중증도와 발생 가능성으로 결합한 개념으로 보았다. $\mathrm{NPSF}$ 는 $\mathrm{RCA}^{2}$ 의 궁극적인 목적이 해저드와 시스템 의 취약성을 규명하여 미래의 위해를 예방함으로써 환자안전을 향상시키기 위한 활동을 수행하는 것이 라고 하였다.

$\mathrm{NPSF}$ 는 사건을 인지한 지 72 시간 이내에 $\mathrm{RCA}^{2}$ 를 시작하도록 하였고 분석에 소요되는 전체 활동 은 30-45일을 넘지 않도록 하며 팀은 4-6명으로 구성하도록 하였다. 본격적으로 $\mathrm{RCA}^{2}$ 를 시작하기 전 해당 사건이 $\mathrm{RCA}^{2}$ 를 수행하기에 적절한 사건인 지 여부를 판단하고 사건에 대해 리스크 기반 우 선순위(risk-based prioritization)를 평가하여 한정 된 자원 안에서 어떤 해저드를 먼저 다루어야할지 결정한다. $\mathrm{RCA}^{2}$ 에서는 이를 위해 $\mathrm{NCPS}$ 와 동일하 게 $\mathrm{SAC}$ 매트릭스를 사용한다. $\mathrm{RCA}^{2}$ 단계는 사건분 석, 활동 수행, 활동 수행 및 효과성 측정, 환류, 리 더십과 위원회의 지지, $\mathrm{RCA}^{2}$ 과정의 효과성 및 지 속가능성 측정으로 이루어져 있다(Figure 1). $\mathrm{RCA}^{2}$ 는 이 단계 중 시스템의 해저드나 취약성을 제거하 거나 조절하기 위한 개선활동 수행 단계가 가장 중 요하다고 하였다.

\section{4. 영국의 런던 프로토콜}

영국의 런던 프로토콜[13]은 1999년에 임페리얼 칼리지 런던(Imperial College London)에서 발표 한 지침이다. 처음 발표 시에는 응급의료분야(acute medical sector)에 집중하였으나 개정판에서는 응급 의료분야뿐만 아니라 정신보건, 앰뷸런스, 일차의료 분야에서도 사용이 가능하도록 확대하였다. 또한 특 이적 기여요인(specific contributory factor)과 일반 적 기여요인(general contributory factor)의 구분을 없앴다. 수집한 자료 기록에 사용되는 양식을 없애 사용하는 기관에서 유연하게 사용할 수 있도록 하면

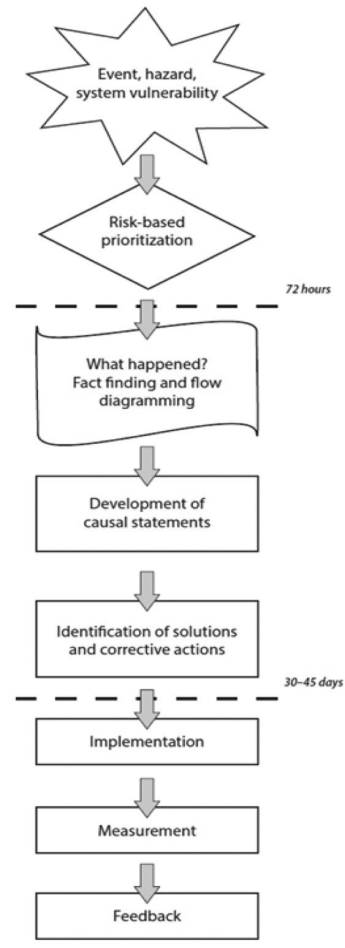

Figure 1. $\mathrm{RCA}^{2}$ process of the National Patient Safety Foundation

Source: National Patient Safety Foundation. $\mathrm{RCA}^{2}$ : Improving Root Cause Analyses and $\mathrm{Ac}^{-}$ tions to Prevent Harm [cited 2017 Mar 15]. Available from: http://www.npsf.org/?page $=\mathrm{RCA}^{2}$.

서 사례를 요약할 때는 표준 양식을 사용하도록 하 고 있다. 런던 프로토콜은 근본원인이라는 용어가 하 나의 원인만 있다고 암시할 가능성이 있는데 실제로 최종 사건의 발생에는 매우 다양한 기여요인이 존재 하기 때문에 이 용어가 적절하지 않다고 보았다. 이 지침에서는 시스템 분석 접근법을 사용하여 근본원 인을 조사하는 것에 그치는 것이 아니라 의료서비스 를 제공하는 체계의 모든 측면에 대해 폭넓게 살펴 보는 것이 중요함을 강조하고 있다. 런던 프로토콜 은 사건의 조사, 분석, 실행을 위한 권고의 과정을 모두 포함하고 있다. 


\section{Review}

\section{근본원인분석 수행을 위한 지침}

런던 프로토콜은 기여요인 유형을 7 가지로 분류 하고 있는데 여기에는 환자 요인, 업무 및 기술 요 인, 개별직원 요인, 팀 요인, 업무환경 요인 외에도 조직 및 관리 요인, 제도적 맥락 요인(institutional context factors)이 있다. 이를 통해 임상적인 요인 뿐만 아니라 제도적인 차원의 요인을 포함하여 분석 할 수 있도록 하고 있다. 이 지침은 의료서비스 제 공 과정에서 발생하는 문제를 표현하기 위해 좀 더 중립적인 용어로써 '의료서비스전달문제(care $\mathrm{de}^{-}$ livery problem, $\mathrm{CDP})^{\prime}$ 를 사용하고 있다. $\mathrm{CDP}$ 는 특 정 행동의 수행이나 누락을 의미하는 것으로써 하나 의 사건(incident)은 여러 개의 $\mathrm{CDP}$ 를 포함할 수 있 다. 런던 프로토콜은 사건 조사 결정, 조사팀 선정, 조직 및 자료 수집, 사건(incident)의 연대기 작성, $\mathrm{CDP}$ 확인, 기여요인 확인, 권고안 작성 및 실행계 획 수립의 7단계를 제시하고 있다. 런던 프로토콜에 는 마지막 단계 안에 실행계획의 효과성 평가가 포 함되어 있기는 하지만 평가를 위한 구체적인 방법에 대한 내용이 부족하며 분석 결과 공유에 대한 구체 적인 지침은 없다.

\section{5. 캐나다의 사건분석틀}

캐나다의 사건분석틀(Incident Analysis Framework)는 캐나다환자안전기구(Canadian Patient Safety Institute), 캐나다의약품안전기구(Institute for Safe Medication Practices Canada) 등 여러 기 관이 협력하여 개발하였다[14]. 이 지침은 환자 및 가족 관점, 사건분석을 위한 다양한 방법, 분석한 내 용이 사건 관리와 어떻게 밀접히 연관되어 있는지에 대한 기술, 기여요인들과 요인들 사이의 관계를 나 타내는 새로운 도식을 제공하고 있다. 이에 더하여 $\mathrm{RCA}$ 후에 활동을 계획하는 방법, 우선순위 정하기, 타당성 확인 및 관리하기에 대한 내용을 개정판에서
새롭게 제공하고 있다.

이 지침은 $\mathrm{RCA}$ 용어 대신 사건분석(incident analysis)이라는 용어를 사용하며 사건분석을 포함 하여 사건이 발생한 이후 취하는 여러 절차와 과 정을 사건관리(incident management)라고 한다. 또 한 세계보건기구에서 개발한 환자안전국제분류체계 (International Classification for Patient Safety)에 따라 환자에게 위해가 발생한 경우에는 유해한 사건 (harmful incident)이라고 하여 기존에 사용하던 유 해사례, 적신호사건, 중대사건(critical incident)의 용 어 대신 사용하고 있다. 환자에게 도달하였으나 뚜 렷한 위해가 발생하지 않은 사건은 무해한 사건(no harm incident), 발생하였으나 환자에게 도달하지 않 은 사건의 경우에는 근접오류라고 하였고 이 세 가 지를 포괄하는 용어로 환자안전사건(patient safety incident)을 사용하고 있다.

특히 이 지침은 사건분석을 소개하기에 앞서 환 자 및 가족의 관점을 수록하면서 환자 및 가족 중 심 의료(patient and family-centered care)를 강 조하고 있다. 이 부분은 캐나다환자안전기구의 환 자 주도 프로그램에 속한 환자와 가족이 작성하였 는데, 환자안전사고가 발생하였을 때 환자 및 가 족과 의료서비스 제공자 간의 동반자 관계(partnership)를 강조하고 있다. 구체적인 내용으로는 발생한 일에 대해 환자에게 설명하고, 유감을 표 하며, 사건이 발생한 과정과 발생 이유를 이해시 켜 주고, 다음 단계에서 일어날 일에 대해 설명하 며, 사실관계 파악 시 참여시키며, 환자/가족 관점 에서 알고 있는 것을 공유할 수 있도록 하는 내용 을 포함하고 있다.

이 지침에서 분석은 시스템 개선에 초점을 맞추 고 있는데 영국의 국가환자안전청(National Patient Safety Agency)에서 개발한 사건결정수(incident decision tree) 모형을 수용하여 해당 사건에 대해 
책무성 검토(accountability review)를 할 것인지 시 스템 개선 검토(system improvement review)를 할 것인지 결정하도록 하였다(Figure 2). 이 지침은 사
건결정수로 판단하였을 때 시스템 개선에 대해 검 토하기로 결정한 사안에 대한 분석 과정을 제시하 고 있다.

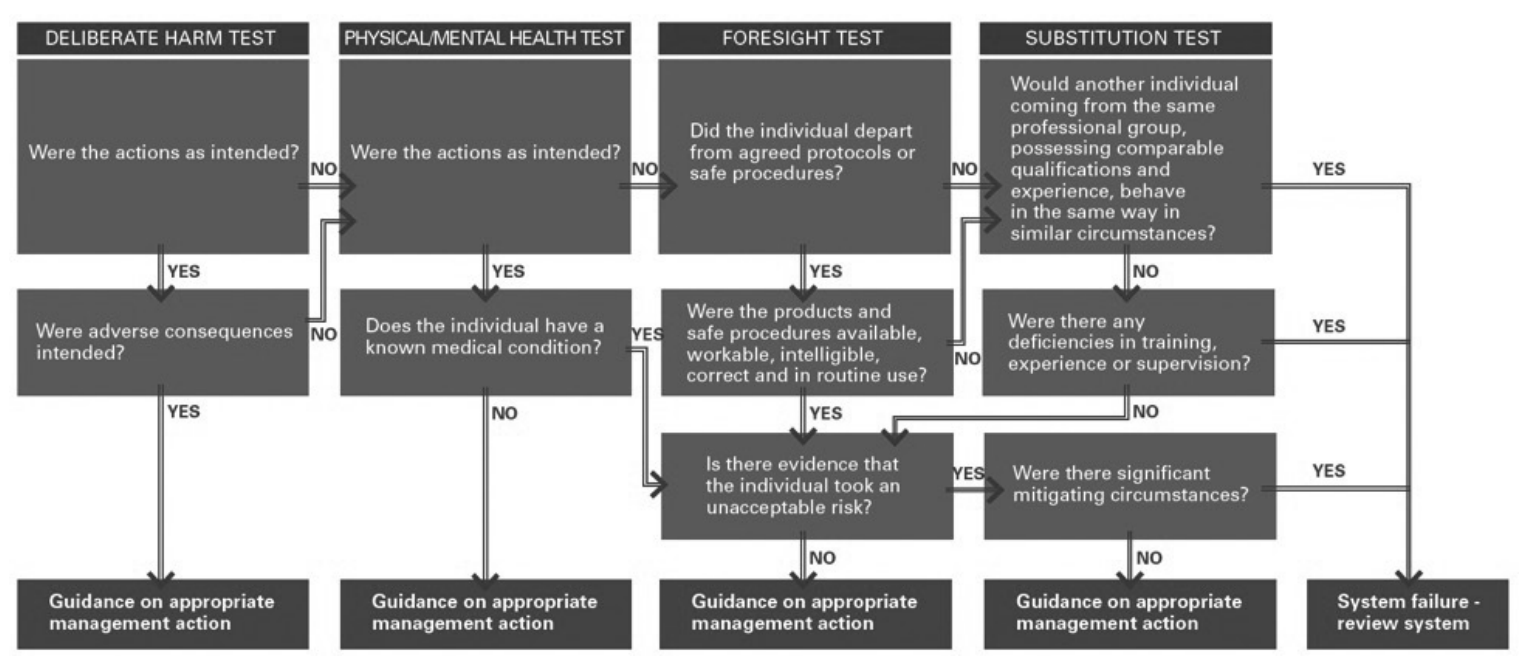

Figure 2. The incident decision tree

Source: Canadian Patient Safety Institute. Incident Analysis [cited 2017 Mar 25]. Available from: http://www.patientsafetyinstitute.ca/en/toolsResources/IncidentAnalysis/Pages/incidentanalysis.aspx.

사건관리는 6 단계로 구분되어 있다. 1 단계는 사건 발생 전부터 시작한다. 사건 발생 전 조직은 사건이 발생하였을 때 이를 처리할 수 있는 절차와 수행 계 획을 가지고 있어야 하며, 이에 대한 리더십과 안전 문화 및 정의문화를 가지고 있어야 한다. 이러한 계 획에 대해서는 검증을 하고 주기적으로 개정하는 것 이 필요하다고 하였다. 2 단계는 사건이 발생한 후에 는 즉각적인 반응을 해야 한다. 여기에는 환자, 가 족, 의료서비스 제공자 등에 대한 돌봄 및 지지, 사 고 보고, 해당 사건에 사용된 의료기기나 약품 등의 확보(secure)가 해당된다. 3 단계는 분석을 위한 준 비 단계로써 기초 조사, 분석 방법 선택, 팀 구성과
팀 접근법의 확인을 포함한다. 4단계는 분석 단계로 써 어떤 일이 발생하였는지, 어떻게 그리고 어떤 이 유로 발생하였는지를 확인하고 개선활동을 수립하는 과정이 있다. 5 단계는 개선활동의 수행, 개선활동의 효과 모니터링 및 평가 단계, 6 단계는 학습한 내용 을 공유하는 단계다.

특히 이 지침은 사건분석 방법을 포괄(comprehensive) 분석, 축약(concise) 분석, 여러 개의 사 건을 분석하는 다사건(multi-incident) 분석으로 분 류하여 각 과정을 소개하고 있으며 사안에 맞게 분석 방법을 선택하여 적용할 수 있도록 하였다 (Table 3). 
Table 3. Criteria for selecting an incident analysis method

\begin{tabular}{|c|c|c|c|}
\hline \multirow[b]{2}{*}{ Criteria } & \multicolumn{3}{|c|}{ Incident analysis methods } \\
\hline & Comprehensive & Concise & Multi-incident \\
\hline Safety assessment code & 3 and some 2 & 1 and some 2 & 1,2 and 3 \\
\hline 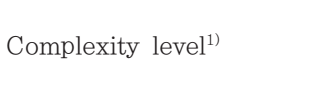 & Complicated, complex & Simple, complicated & $\begin{array}{l}\text { Simple, complicated } \\
\text { or complex }\end{array}$ \\
\hline Area of impact & $\begin{array}{l}\text { Team, unit/program, } \\
\text { organization, system }\end{array}$ & $\begin{array}{l}\text { Team, unit/program, } \\
\text { possible organization }\end{array}$ & $\begin{array}{c}\text { Team, unit/program, } \\
\text { organization, system, } \\
\text { sector, industry }\end{array}$ \\
\hline $\begin{array}{l}\text { Context - internal and } \\
\text { external pressures }\end{array}$ & High & Low & Low, medium or high \\
\hline $\begin{array}{l}\text { Resources required/ } \\
\text { available }^{2)}\end{array}$ & Moderate to extensive & Limited & Moderate to extensive \\
\hline Timelines & Weeks to months & Hours to days & Variable \\
\hline
\end{tabular}

1) Degree of agreement, certainty and number of interactions

2) Time, financial and human

Source: Canadian Patient Safety Institute. Incident Analysis [cited 2017 Mar 25]. Available from: http://www.patientsafetyinstitute.ca/en/toolsResources/IncidentAnalysis/Pages/incidentanalysis.aspx.

\section{6. 일본의 아임세이퍼}

아임세이퍼(ImSAFER)는 Improvement for Medical System by Analyzing Fault root in human ERror incident의 약자이다. 아임세이퍼는 인간행동 모델을 이용하여 인간의 오류 행동 및 이와 관계된 사건과 현상을 분석해 나가는 방법으로써 일본의 가 와노 류타로가 제안하였다[15]. 이 지침에서는 인적 오류란 인간이 가진 특성과 인간을 둘러싼 환경이 상호작용하면서 결정한 행동 중에서 기대하는 범위 를 벗어난 것으로써 원인이 아니라 결과라고 설명한 다. 여기서 인간의 특성은 생리학적, 심리학적, 인지 적 특성을 말하며 환경은 인간을 둘러싼 기계, 순서 설명서, 팀, 교육시스템 등을 말한다. 인적 오류를 방
지하기 위해서는 공학 분야에서 유래한 인적 요소를 사용하는 것이 효과적인 방법인데, 인간에게 주의를 환기시키는 것만으로는 사고 재발을 방지할 수 없으 며 시스템을 파악하는 것이 인적 오류 방지에 중요 하다고 하였다. 아임세이퍼는 분석 절차마다 사용할 수 있는 도구를 제시하고 있으며 개선안을 도출하는 과정도 포함하고 있다.

아임세이퍼는 분석, 개선, 실시, 평가로 진행되는 7단계 절차로 구성되어 있다. 우선 분석을 위한 사전 준비 단계에서는 분석 사례에 관한 정보를 수집하고 분석에 참여하는 팀 멤버를 구성하며 분석에 필요한 도구를 준비한다. 분석 단계에서는 시계열사상관련 도를 그리며 이 그림에서 문제점을 찾고 배후요인을 탐색한다. 아임세이퍼의 특징적인 점은 시간의 흐름 
에 따라 일어난 사건과 현상에 관한 그림인 시계열 사상관련도를 작성하는 것이다. 시계열사상관련도는 사건과 현상을 수직으로 배치하여 화살표로 연결하 고 이에 관련된 의료인, 환자, 보호자 등의 관계자를 상단에 수평으로 표시하여 사건의 흐름과 관련 당사 자를 한 눈에 볼 수 있도록 도식화하는 도구이다. 개 선 단계에서는 가능한 개선책을 열거하고 실행 가능 한 개선책을 결정한다. 실시 단계에서는 개선책을 실 행하고 평가 단계에서는 실행한 개선책을 평가한다.

지금까지 소개한 근본원인분석 수행을 위한 지침 을 요약하면 부록과 같다.

\section{III. 결론}

이 글에서는 국외의 $\mathrm{RCA}$ 수행 지침을 살펴보았 다. 지침 중에는 NCPS 지침과 같이 국가 기관에서 운영하는 보고시스템에서 사용할 수 있는 지침도 있 었고, 영국의 런던 프로토콜처럼 구체적인 양식을 따로 제공하지 않고 유연하게 수행할 수 있도록 제 시한 지침도 있었다. $\mathrm{TJC}$ 지침은 $\mathrm{RCA}$ 수행에 필요 한 많은 단계를 제시하면서 상세한 지침을 제공하 기도 하였고 캐나다의 사건분석틀이나 일본의 아임 세이퍼는 사건에 따라 간략한 단계로 수행할 수 있 는 $\mathrm{RCA}$ 절차를 제시하기도 하였다. $\mathrm{RCA}$ 수행 단계 의 구분이나 지침에서 제공하는 도구 등에서 차이가 있었지만 전체적인 수행 순서는 유사하였고, 개인의 잘못을 찾아서 처벌하는 것이 목적이 아니라 시스템 의 취약성을 찾아 개선하여 향후 재발 방지를 목적 으로 하는 점에서 공통점이 있었다. 그래서 각 지침 에서는 사건의 분석뿐만 아니라 시스템 개선을 위한 활동 단계를 세밀하게 제시하고 있으며 활동 수행 후 이에 대한 효과 평가도 포함하고 있다.
살펴본 바와 같이 국외에서는 여러 $\mathrm{RCA}$ 지침을 개발하여 사용하고 있으나 국내에서는 $\mathrm{RCA}$ 지침 이 개발된 사례가 없어 국외의 RCA 지침을 번역하 여 사용하고 있다. 그러나 국내 연구에 따르면 아직 까지 많은 의료기관에서 $\mathrm{RCA}$ 에 대한 지식 부족으 로 $\mathrm{RCA}$ 수행에 어려움을 겪는 것으로 나타나고 있 다. 이 글에서 검토한 다양한 $\mathrm{RCA}$ 지침을 의료기관 에서 $\mathrm{RCA}$ 수행 시 활용할 수 있을 것이며, 향후 국 내의 $\mathrm{RCA}$ 지침 개발 시 기초자료로 이용할 수 있 을 것이다.

\section{IV. 참고문헌}

1. Patient Safety Act Article 2, Enforcement Rule of Patient Safety Act Article 2.

2. Lee SI. Approaches to improve patient safety in healthcare organizations. Journal of the Korean Medical Association. 2015;58(2):90-92.

3. de Vries EN, Ramrattan MA, Smorenburg SM, Gouma DJ, Boermeester MA. The incidence and nature of in-hospital adverse events: a systematic review. Quality \& Safety in Health Care. 2008;17:216-223.

4. National Health Insurance Service, Health Insurance Review \& Assessment Service. 2015 National Health Insurance Statistical Yearbook. Wonju, Korea; National Health Insurance Service, Health Insurance Review \& $\mathrm{As}^{-}$ sessment Service; 2015.

5. Lee SI. Development of institutional mechanism for improving patient safety in Korea. Osong, Korea: Korean Centers for Disease Control and Prevention; 2013. 


\section{Review}

\section{근본원인분석 수행을 위한 지침}

6. Shin HH. Investigation and promotion of $\mathrm{pa}^{-}$ tient safety actions at medical institutions. Seoul, Korea: Korean Institute of Hospital Management; 2015.

7. Ministry of Health and Welfare - Korean Institute for Healthcare Accreditation. 2016 the Act on Patient Safety practice manual. Seoul, Korea: Korean Institute for Healthcare Accreditation; 2016.

8. Jung YY. Establishment and operation plan of patient safety incident management system. Seoul, Korea: Korean Institute for Healthcare Accreditation; 2014.

9. Wu AW, Lipshutz AK, Pronovost PJ. Effectiveness and efficiency of root cause analysis in medicine. The Journal of the American Medical Association. 2008;299(6):685-687.

10. Parker J. editor. The Joint commission. Root Cause Analysis in Health Care: Tools and Techniques. 5th ed. Illinois, USA: Oak Brook; 2015.

11. VA National Center for Patient Safety. Root Cause Analysis [cited 2017 Apr 10]. Available from: https://www.patientsafety.va.gov/ professionals/onthejob/rca.asp.

12. National Patient Safety Foundation. RCA2: Improving Root Cause Analyses and Actions to Prevent Harm [cited 2017 Mar 15]. Available from: http://www.npsf.org/?page=RCA2 .

13. Imperial College London. Systems Analysis of Clinical Incidents: The London Protocol [cited 2017 Apr 20]. Available from: http://www. imperial.ac.uk/patient-safety-translational-research-centre/education/training-ma- terials-for-use-in-research-and-clinical-practice/the-london-protocol/.

14. Canadian Patient Safety Institute. Incident Analysis [cited 2017 Mar 25]. Available from: http://www.patientsafetyinstitute.ca/en/toolsResources/IncidentAnalysis/Pages/incidentanalysis.aspx.

15. Kawano R. Improvement for medical System by Analyzing Fault root in human ERror incident. Lee MJ, translator. Seoul, Korea: $\mathrm{Ha}-$ nEon; 2014. 
이현정, 최은영, 옥민수, 이상일

부록. 근본원인분석 지침

\begin{tabular}{|c|c|c|c|c|c|c|}
\hline & $\begin{array}{c}\text { 더 조인트 커미션의 } \\
\text { 지침서 }\end{array}$ & $\begin{array}{c}\text { 국가환자안전센터의 } \\
\text { 지침서 }\end{array}$ & $\begin{array}{c}\text { 환자안전재단의 } \\
\text { 근본원인분석과 행동 }\end{array}$ & $\begin{array}{c}\text { 임페리얼 칼리지 런던의 } \\
\text { 런던 프로토콜 }\end{array}$ & $\begin{array}{c}\text { 캐나다환자안전기구 } \\
\text { 등의 사건분석틀 }\end{array}$ & 아임세이퍼 \\
\hline 국가 & 미국 & 미국 & 미국 & 영국 & 캐나다 & 일본 \\
\hline $\mathrm{RCA}^{1)}$ & · 팀 구성 & - RCA 수행 여 & - 사건분석 & - 사건 조사 결정 & - 사건 발생 전 리더 & - 시계열사상관련 \\
\hline \multirow[t]{38}{*}{ 단계 } & · 문제 정의 & 부 결정 & 활동 수행 & · 조사팀 선정 & 십 지원, 안전하고 & 도 작성 \\
\hline & · 문제 연구 & 필요한 경우 즉각 & 활동 수행 및 효과 & · 조직 및 자료 수집 & 공정한 문화, 사건 & · 문제점 찾기 \\
\hline & - 무엇이 일어났는 & 적 행동 수행 & 성 측정 & - 사건의 연대기 & 관리 계획 수립 & - 배후 요인 탐색 \\
\hline & 지 확인 & - 선언문 작성 및 & 환류 & 작성 & - 사건 발생 시 즉각 & - 개선책 열거 \\
\hline & - 절차적 기여요 & 팀 구성 & · 리더십과 위원회의 & - $\mathrm{CDP}^{2)}$ 확인 & 적 반응 & - 실행 가능한 개선 \\
\hline & 인 확인 & ·팀 훈련 & 지지 & · 기여요인 확인 & - 사건분석을 위 & 책 결정 \\
\hline & · 다른 기여요인 & - 사건순서도 초 & $\mathrm{RCA}$ 과정의 효과 & - 권고안 작성 및 & 한 준비 & · 개선책 실행 \\
\hline & 확인 & 안 작성 & 성 및 지속가능 & 실행 계획 수립 & - 사건분석 & · 실행한 개선책 \\
\hline & · 근접원인과 기반원 & · 부족한 정보 확인 & 성 측정 & & - 개선활동 수행 및 & 평가 \\
\hline & 인에 대한 데이터 & · 필요한 정보 구 & & & 효과 모니터링 & \\
\hline & 수집 및 사정 & 체화 & & & - 학습한 내용의 & \\
\hline & . 임시변화의 설계 & · 탐색 질문, 면접 & & & 공유 & \\
\hline & 및 도입 & 도구 사용 & & & & \\
\hline & · 관련 시스템 확인 & · 자료수집 통한 사 & & & & \\
\hline & - 근본원인 목록 추 & 실 확인 & & & & \\
\hline & 리기 & - 정보 합성 & & & & \\
\hline & · 근본원인 확인 및 & - 사건순서도 & & & & \\
\hline & 상호관계 강조 & 최종 확정 & & & & \\
\hline & - 위험성 감소전략 & ·근본원인 및 기여 & & & & \\
\hline & 탐색 및 규명 & 요인 확인 & & & & \\
\hline & · 개선활동 개발 & - 과거 유사 RCA & & & & \\
\hline & · 제안된 개선활 & 개선활동 확인 & & & & \\
\hline & 동 평가 & · 활동 계획 수립 & & & & \\
\hline & . 설계 개선 & - 사건 보고자에게 & & & & \\
\hline & 활동 계획 보장 & 개선활동 알림 & & & & \\
\hline & · 개선 계획 도입 & RCA 결과와 권고 & & & & \\
\hline & - 효과적 척도 개발 & 사항 작성 및 결과 & & & & \\
\hline & ·도입과 개선 노력 & 공유 계획 & & & & \\
\hline & 에 대한 평가 & · 관리자에게 $\mathrm{RCA}$ & & & & \\
\hline & · 추가 행동 & 결과 제출 & & & & \\
\hline & · 결과의 전달 & - 보고 문서 작성 & & & & \\
\hline & & 완료 & & & & \\
\hline & & - 소요 비용, 인당 & & & & \\
\hline & & 시간 산정 & & & & \\
\hline & & - $\mathrm{RCA}$ 및 활동 계 & & & & \\
\hline & & 획에 대한 최종 & & & & \\
\hline & & 서명 & & & & \\
\hline & & 활동의 효과 보고 & & & & \\
\hline
\end{tabular}




\begin{tabular}{|c|c|c|c|c|c|c|}
\hline & $\begin{array}{c}\text { 더 조인트 커미션의 } \\
\text { 지침서 }\end{array}$ & $\begin{array}{c}\text { 국가환자안전센터의 } \\
\text { 지침서 }\end{array}$ & $\begin{array}{c}\text { 환자안전재단의 } \\
\text { 근본원인분석과 행동 }\end{array}$ & $\begin{array}{c}\text { 임페리얼 칼리지 런던의 } \\
\text { 런던 프로토콜 }\end{array}$ & $\begin{array}{c}\text { 캐나다환자안전기구 } \\
\text { 등의 사건분석틀 }\end{array}$ & 아임세이퍼 \\
\hline 국가 & 미국 & 미국 & 미국 & 영국 & 캐나다 & 일본 \\
\hline 특징 & $\begin{array}{l}\text { ·적신호사건의 } \\
\mathrm{RCA} \text { 에 초점을 맞 } \\
\text { 춘 지침서 } \\
\text { · 각 단계마다 사용 } \\
\text { 할 수 있는 도구 } \\
\text { 및 활용 가능한 문 } \\
\text { 서 양식 제공 } \\
\text { · } \mathrm{RCA} \text { 를 적용한 구 } \\
\text { 체적 사례 제공 }\end{array}$ & $\begin{array}{l}\text { · 사건 평가 도구인 } \\
\mathrm{SAC}^{3)} \text { 매트릭스를 } \\
\text { 사용하여 사건의 } \\
\text { 위험 분류 }\end{array}$ & $\begin{array}{l}\text { - 시스템의 해저드나 } \\
\text { 취약성을 제거하거 } \\
\text { 나 조절하기 위한 } \\
\text { 개선활동 수행 단 } \\
\text { 계 강조 }\end{array}$ & $\begin{array}{l}\text { 기여요인의 유형 } \\
\text { 분류 제공 }\end{array}$ & $\begin{array}{l}\text { 환자 및 가족의 관 } \\
\text { 점을 제공하면서 } \\
\text { 환자 및 가족 중심 } \\
\text { 의료 강조 } \\
\text { · 사건결정수 모형 } \\
\text { 을 사용하여 사 } \\
\text { 건 분류 }\end{array}$ & $\begin{array}{l}\text {. 인적 오류를 방지 } \\
\text { 하기 위해서 공학 } \\
\text { 분야에서 유래한 } \\
\text { 인적 요소 이용 } \\
\text { 분석 절차마다 사 } \\
\text { 용할 수 있는 도구 } \\
\text { 및 개선안 도출 과 } \\
\text { 정 제시 }\end{array}$ \\
\hline $\begin{array}{l}\text { 활용 시 } \\
\text { 장점 및 } \\
\text { 고려사항 }\end{array}$ & $\begin{array}{l}\text { ·지침서에서 도 } \\
\text { 구 및 문서 양식 } \\
\text { 을 구체적으로 제 } \\
\text { 시하므로 이를 활 } \\
\text { 용한 RCA 수행이 } \\
\text { 가능함 } \\
\text { · RCA 단계가 } 21 \text { 단 } \\
\text { 계로 세분화되어 } \\
\text { 있어 해당 의료기 } \\
\text { 관 및 RCA 담당 } \\
\text { 부서의 상황에 맞 } \\
\text { 게 수정하여 사용 } \\
\text { 하는 것이 필요함 }\end{array}$ & $\begin{array}{l}\mathrm{RCA} \text { 과정에서 사 } \\
\text { 용할 수 있는 질 } \\
\text { 문, 근본원인진술 } \\
\text { 문 작성 규칙, 활 } \\
\text { 동 계획 개발 과 } \\
\text { 정, 평가지표 선택 } \\
\text { 과정 등을 활용할 } \\
\text { 수 있음 }\end{array}$ & $\begin{array}{l}\text { 지침서에서 제 } \\
\text { 공하는 SAC 매 } \\
\text { 트릭스, 탐색질 } \\
\text { 문(triggerin-g } \\
\text { question), 인터 } \\
\text { 뷰 방법 등 활용 } \\
\text { 가능함 }\end{array}$ & $\begin{array}{l}\text { · 기여요인에 제도적 } \\
\text { 맥락 요인을 포함 } \\
\text { 하고 있어서 기여 } \\
\text { 요인에 대한 폭넓 } \\
\text { 은 시각을 제공함 } \\
\text { · RCA 시 활용 가 } \\
\text { 능한 구체적 양 } \\
\text { 식을 제시하지 않 } \\
\text { 고 있어서 다른 지 } \\
\text { 침과 병행 사용 } \\
\text { 필요함 }\end{array}$ & $\begin{array}{l}\text { · 사건의 특성에 따 } \\
\text { 라 분석 방법을 } \\
\text { 선택하여 활용할 } \\
\text { 수 있음 }\end{array}$ & $\begin{array}{l}\text { · 시계열사상관련 } \\
\text { 도 작성법과 이를 } \\
\text { 바탕으로 한 문제 } \\
\text { 점 발견, 배후요 } \\
\text { 인 탐색 방법을 구 } \\
\text { 체적으로 제시하 } \\
\text { 고 있음 } \\
\text { · 수행 단계를 간 } \\
\text { 략하게 만든 } \\
\text { 'QuickSAFER' 를 } \\
\text { 제시하고 있음 }\end{array}$ \\
\hline
\end{tabular}

1) RCA root cause analysis 근본원인분석; 2) CDP care delivery problem 의료서비스전달문제; 3) SAC safety assessment code 안전평가코드 